\title{
BMJ Open CRISTAL: protocol for a cluster randomised, crossover, non-inferiority trial of aspirin compared to low molecular weight heparin for venous thromboembolism prophylaxis in hip or knee arthroplasty, a registry nested study
}

\author{
Verinder Singh Sidhu (10 , ${ }^{1}$ Steven E Graves, ${ }^{2}$ Rachelle Buchbinder, ${ }^{3,4}$ \\ Justine Maree Naylor, ${ }^{1}$ Nicole L Pratt, ${ }^{5}$ Richard S de Steiger, ${ }^{6}$ Beng H Chong, ${ }^{7}$ \\ Ilana N Ackerman (D) , ${ }^{3}$ Sam Adie, ${ }^{8}$ Anthony Harris, ${ }^{9}$ Amber Hansen, ${ }^{1}$ \\ Maggie Cripps, ${ }^{1}$ Michelle Lorimer, ${ }^{10}$ Steve Webb, ${ }^{11,12}$ Ornella Clavisi, ${ }^{13}$ \\ Elizabeth C Griffith, ${ }^{10}$ Durga Anandan, ${ }^{2}$ Grace O'Donohue, ${ }^{2}$ Thu-Lan Kelly, ${ }^{5}$ \\ Ian A Harris ${ }^{1,14}$
}

To cite: Sidhu VS, Graves SE, Buchbinder R, et al. CRISTAL: protocol for a cluster randomised, crossover, noninferiority trial of aspirin compared to low molecular weight heparin for venous thromboembolism prophylaxis in hip or knee arthroplasty, a registry nested study. BMJ Open 2019;9:e031657. doi:10.1136/ bmjopen-2019-031657

- Prepublication history and additional material for this paper are available online. To view these files, please visit the journal online (http://dx.doi. org/10.1136/bmjopen-2019031657).

Received 15 May 2019 Revised 19 September 2019 Accepted 07 October 2019

D) Check for updates

(c) Author(s) (or their employer(s)) 2019. Re-use permitted under CC BY-NC. No commercial re-use. See rights and permissions. Published by BMJ.

For numbered affiliations see end of article.

Correspondence to Dr Verinder Singh Sidhu; verinder.s.sidhu@gmail.com

\section{ABSTRACT}

Introduction Venous thromboembolism (VTE) is a serious complication following hip arthroplasty $(\mathrm{HA})$ and knee arthroplasty (KA). This study aims to determine whether aspirin is non-inferior to low molecular weight heparin (LMWH) in preventing symptomatic VTE following HA and KA.

Methods and analysis This is a cluster randomised, crossover, non-inferiority, trial nested within the Australian Orthopaedic Association National Joint Replacement Registry (AOANJRR). The clusters will consist of Australian hospitals performing at least $250 \mathrm{HA}$ and/or KA procedures per annum. All adult patients undergoing HA or KA will be included. The intervention will be aspirin, orally, 85$150 \mathrm{mg}$ daily. The comparator will be LMWH (enoxaparin) $40 \mathrm{mg}$, subcutaneously, daily. Both drugs will commence within 24 hours postoperatively and continue for 35 days after HA and 14 days after KA. Each hospital will be randomised to commence with aspirin or LMWH and then crossover to the alternative treatment after meeting the recruitment target. Data will be collected through the AOANJRR via patient-reported surveys. The primary outcome is symptomatic VTE within 90 days post surgery, verified by AOANJRR staff. The primary analysis will include only patients undergoing elective primary total hip arthroplasty and total knee arthroplasty for osteoarthritis. Secondary outcomes will include symptomatic VTE for all $\mathrm{HA}$ and KA (including partial and revision) within 90 days, readmission, reoperation, major bleeding and death within 90 days and reoperation, death and patient-reported pain, function and health status at 6 months. If aspirin is found to be inferior, a cost-effectiveness analysis will be conducted. The study will aim to recruit 15562 patients from 31 hospitals.
Strengths and limitations of this study

- Registry-nested trial allowing efficient data collection and large sample size.

- Crossover design balancing potential clusterdependent confounders.

- Use of outcome and compliance verification processes.

- Relies on patient reporting of primary outcome.

- Does not measure asymptomatic (subclinical) venous thromboembolism.

Ethics and dissemination Ethics approval has been granted. Trial results will be submitted for publication. The trial is registered with the Australian New Zealand Clinical Trials Registry (ACTRN12618001879257, preresults) and is endorsed by the Australia and New Zealand Musculoskeletal Clinical Trials Network.

\section{INTRODUCTION}

Over 100000 hip and knee arthroplasty (HA, $\mathrm{KA}$ ) procedures are performed each year in Australia. ${ }^{1}$ Symptomatic postoperative venous thromboembolism (VTE), comprising deep venous thrombosis (DVT) and pulmonary embolus (PE), may occur in approximately $2 \%$ of patients. ${ }^{2}$ Apart from mechanical prophylaxis (compression pumps or stockings and early mobilisation), patients undergoing $\mathrm{HA}$ and KA also receive chemoprophylaxis for VTE prevention, with most patients in 
Australia receiving either low molecular weight heparin (LMWH) or aspirin. ${ }^{3}$

Guideline recommendations for VTE prophylaxis vary due to a lack of evidence regarding the comparative safety and effectiveness of these two common chemoprophylaxis agents. ${ }^{4-7}$ Aspirin is a low cost, over-the-counter, safe medication that is easy to take (one tablet taken orally, daily). LMWH requires daily injection (often requiring professional or family support), is more expensive and requires a prescription, but has a larger body of evidence confirming its effectiveness. ${ }^{8-11}$

Current practice guidelines provide conflicting recommendations for VTE prophylaxis. The National Institute of Health and Care Excellence guidelines (UK, 2018) now recommend using LMWH, aspirin or novel oral anticoagulants (NOACs) for VTE prophylaxis following KA and recommend either LMWH or NOACs following HA, with aspirin only recommended for extended prophylaxis. Aspirin was not recommended in the previous version of this guideline. ${ }^{6}$ In the USA, separate guidelines have been produced by the American College of Chest Physicians and the American Association of Orthopaedic Surgeons. ${ }^{45}$ Both recommend the use of LMWH, NOACs or aspirin. The Australian National Health and Medical Research Council guidelines (2011) did not recommend aspirin, however these guidelines were rescinded in 2016 as they were considered outdated. ${ }^{12}$

The current literature also provides conflicting evidence. Few systematic reviews have synthesised the evidence for aspirin, despite international guideline recommendations and its common use for prevention of VTE following HA and KA. ${ }^{113-18}$ Two recent small systematic reviews included data from six small trials (with sample sizes ranging from 121 to 1728 patients) using aspirin as a comparator, and concluded that larger trials are needed. ${ }^{19}{ }^{20}$ In both reviews, the evidence was dominated by one trial of 778 patients comparing aspirin with LMWH in total hip arthroplasty (THA) ${ }^{21}$ This trial was stopped early due to poor recruitment and all patients received LMWH for the first 10 days before random allocation to aspirin or continued LMWH. Another five trials were also described, including a total of 936 patients, but these trials were small, measured different outcomes and were at risk of bias. ${ }^{19}$ Subsequent to the above systematic reviews, a large randomised trial (including 3424 patients) has been published and concluded aspirin was non-inferior to rivaroxaban (a NOAC).$^{22}$ However, both groups were treated with rivaroxaban for the first 5 days before being randomised to aspirin or rivaroxaban for the following 2-4 weeks. This does not reflect the way that aspirin is used in Australia, where it is usually commenced within 24 hours of surgery and not given in combination with any other VTE chemoprophylaxis. ${ }^{3}$

While studies using administrative datasets should be interpreted with caution due to risk of observer bias and incomplete data, three studies of aspirin using large administrative datasets have been reported. ${ }^{23-25}$ The first, from the USA, used data from 93804 patients undergoing elective total knee arthroplasty (TKA) to compare outcomes for patients receiving three different forms of VTE prophylaxis: injectable prophylaxis (LMWH or fondaparinux), aspirin or warfarin. No difference was found in the mortality rates or rates of postoperative bleeding complications between the three groups, and there was no difference in the rate of VTE comparing LMWH with aspirin. ${ }^{23}$ Two studies using data from the National Joint Registry for England, Wales, Northern Ireland and the Isle of Man for patients undergoing THA and TKA with over 200000 patients showed no difference in VTE rates or re-operations (up to 90 days) between LMWH and aspirin groups. However, the reported rates of VTE were very low, possibly due to underdetection. ${ }^{24} 25$

\section{Study rationale}

The existing uncertainty within the available literature and inconsistencies in available clinical practice guidelines likely contribute to the widespread practice variation currently seen in Australia. ${ }^{326}$ There has been a trend towards declining LMWH use over the last decade and a shift towards aspirin-based protocols, although high-level evidence remains lacking. ${ }^{3}$ Aspirin does not require a prescription, is easier to achieve adherence (tablet rather than injection), is safe and is inexpensive. High-quality evidence is required to determine the clinical effectiveness, cost-effectiveness and safety of aspirin compared with LMWH for VTE prophylaxis after HA and KA.

\section{Objectives}

\section{Primary objective}

The primary objective of this study is to determine whether aspirin is non-inferior to LMWH in the prevention of symptomatic VTE (either DVT or PE) occurring within 90 days in adults undergoing primary, elective THA or TKA for osteoarthritis (OA), who are not currently on anticoagulant medication for a pre-existing condition (specifically warfarin, NOACs or dual antiplatelet therapy).

\section{Secondary objectives}

1. To determine whether aspirin is non-inferior to LMWH for THA and TKA separately, unilateral and bilateral arthroplasty and subgroups of VTE (below-knee DVT, above-knee DVT and PE as separate outcomes).

2. To determine whether aspirin is non-inferior to LMWH in preventing VTE for all patients undergoing HA or KA (for any indication, including partial and revision arthroplasty).

3. To compare safety outcomes (death, reoperation, readmission and major bleeding events within 90 days and reoperation and death within 6 months, and reasons for these) between LMWH and aspirin groups.

4. To compare patient-reported pain, function and health status at 6 months between the two treatment groups (specifically the Oxford Hip and Knee Scores, ${ }^{27}$ the EuroQol EQ-5D and EuroQol-visual analogue scale (EQ-VAS) scores $^{28}$ and a $0-10$ joint pain score). 
5. To perform a cost-effectiveness analysis comparing aspirin and LMWH, if aspirin is found to be inferior to LMWH.

6. To compare rates of persistent wound drainage (PWD) and wound disturbance between aspirin and LMWH groups as a substudy.

\section{METHODS AND ANALYSIS CRISTAL study design}

CRISTAL (a cluster randomised, non-inferiority trial of aspirin compared to low molecular weight heparin for venous thromboembolism prophylaxis in hip or knee arthroplasty, a registry nested study) has been designed as a pragmatic, non-inferiority, cluster-randomised, crossover trial and will be nested within the Australian Orthopaedic Association National Joint Replacement Registry (AOANJRR). The AOANJRR was established in 1999 and reports on demographic data, revision surgery and mortality after joint arthroplasty in Australia, with near complete $(98 \%-100 \%)$ national coverage. ${ }^{1}$ The AOANJRR has established an online Clinical Trials Platform to allow direct data capture by patients preoperatively and postoperatively. The CRISTAL trial will use this platform.

Thirty-one hospitals across Australia have agreed to participate. Patient recruitment began on 15 April 2019 and is planned to be complete by 31 August 2021. Figure 1 demonstrates a flow sheet of the study design.

\section{Eligibility criteria}

At a hospital level, all hospitals performing $\mathrm{HA}$ and KA procedures in Australia are eligible providing that the hospital department (or surgeon group within the hospital) agrees to participate in the study, agrees to adhere to the study protocol throughout the course of trial and that the hospital or surgeon group performs at least $250 \mathrm{HA}$ and/or KA procedures per annum. This number has been chosen based on the power calculation for this study. The chief investigator (IAH) was responsible for recruiting hospital departments.

At a patient level, all adult patients (aged 18 years and older) undergoing primary, revision or partial HA or KA for any indication will be eligible for the trial; there are no patient exclusion criteria as the trial is considered a pragmatic study and recruitment and randomisation will occur at hospital level, not at the level of the individual patient. Each recruited hospital will be responsible for recruiting patients and the recruitment of patients will be tracked by the AOANJRR using the Clinical Trials Platform.

\section{Rationale for study design}

A cluster-randomised, crossover trial will allow recruitment of all available patients, without excessive loss of potentially eligible patients. An individually randomised non-inferiority trial was contemplated, however, individually consenting patients would require an additional process that would render entry into the study more

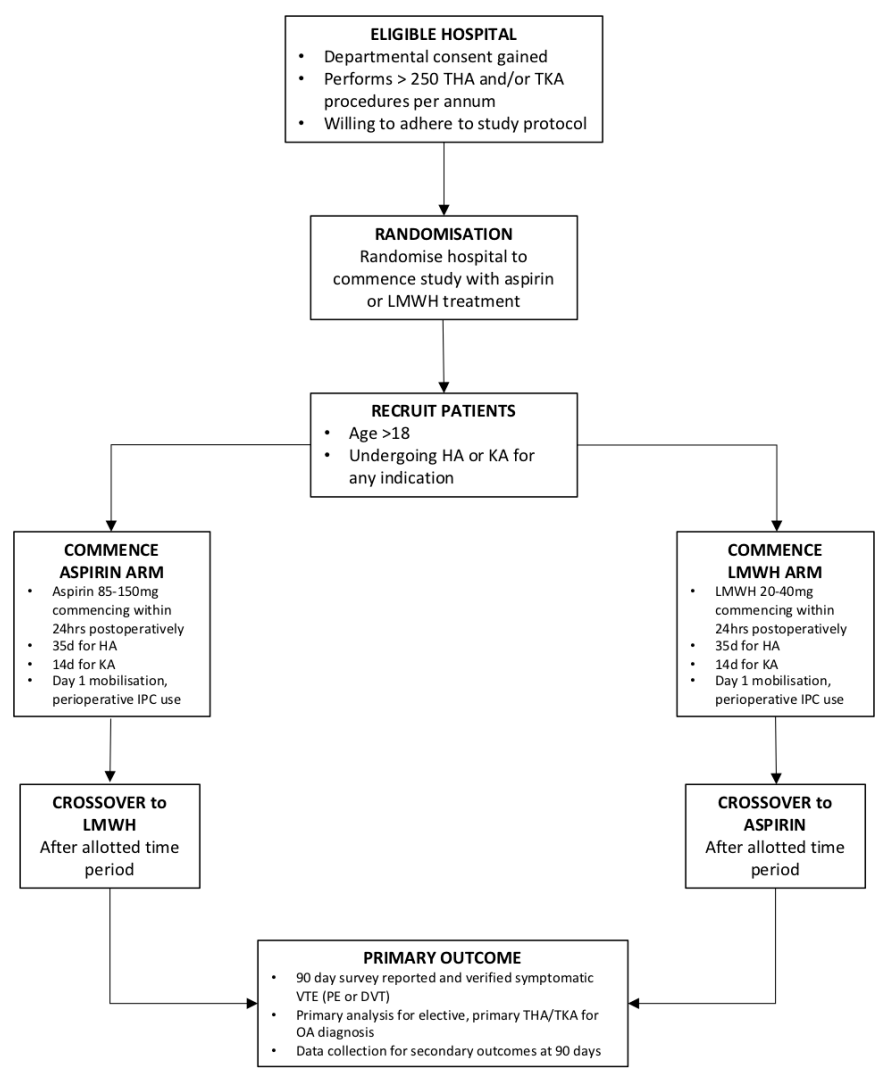

Figure 1 Study design flow sheet. DVT, deep venous thrombosis; HA, hip arthroplasty; IPC, intermittent pneumatic compression; KA, knee arthroplasty; LMWH, low molecular weight heparin; OA, osteoarthritis; PE, pulmonary embolus; THA, total hip arthroplasty; TKA, total knee arthroplasty; VTE, venous thromboembolism.

burdensome for patients and sites, potentially leading to a lower proportion of eligible patients being recruited. Given the high numbers of patients required for the trial (sample size calculation given below) and that both interventions are commonly used and acceptable forms of VTE prophylaxis in Australia, this trial design was chosen as the most efficient, pragmatic method, without exposing patients to undue risk or burden in trial participation. The inclusion of patients who have been traditionally excluded from similar trials (eg, patients with a prior VTE history not currently on anticoagulant therapy, revision and partial arthroplasty) ${ }^{2122}$ will further reduce the potential for confusion as to which patients may or may not be eligible for trial inclusion and reflects the pragmatic nature of the study. It also enhances the likelihood that the trial findings will be later adopted into clinical practice.

The crossover design was chosen as an additional feature to allow important potential confounders (such as differences in socioeconomic status and patient management between clusters) to be balanced between treatment groups. ${ }^{29} 30$

\section{Treatment groups and randomisation}

Each hospital will be randomised to two consecutive periods of a standard protocol of LMWH and a standard 
protocol of aspirin as VTE prophylaxis. Patients in the aspirin group will receive aspirin at a dose of $85-150 \mathrm{mg}$ once daily, orally for 35 days post HA and for 14 days post KA commencing within 24 hours of surgery. Patients in the LMWH group will receive enoxaparin at a dose of $40 \mathrm{mg}$ once daily, subcutaneously for the same time periods. The dose of LMWH will be reduced to $20 \mathrm{mg}$ for patients who weigh $<50 \mathrm{~kg}$ and for patients with an estimated glomerular filtration rate of $<30 \mathrm{~mL} / \mathrm{min}$ who are not on dialysis. These doses and time periods have been chosen as they reflect the best current available evidence and guidelines for VTE prophylaxis. ${ }^{4621} 22$ Other interventions that will be standard across all sites are the intraoperative and postoperative use of intermittent pneumatic compression (IPC) calf devices until patients are mobile, the use of compression stockings and mobilisation offered on day 0 or day 1 postoperatively.

All patients at participating hospitals undergoing any HA or KA procedure (total, partial or revision arthroplasty for any indication including fracture) will receive the allocated study drug, unless they are already taking anticoagulant medication for a pre-existing condition (specifically warfarin, NOACs or dual antiplatelet therapy) or have a contraindication to aspirin or LMWH. These patients will have these data recorded preoperatively and will be managed according to local site peri-operative protocols; they will not be excluded from the trial but will not be included in the primary analysis. Patients taking single antiplatelet therapy for a pre-existing condition will be given the study drug as VTE prophylaxis and will be included in the primary analysis. Figure 2 demonstrates a patient management flow sheet.

Hospitals will be randomised to commence with either LMWH or aspirin, in blocks of size four by statisticians from the South Australian Health and Medical Research Institute, independent of study investigators. Study investigators will be blinded to group allocation. Hospitals will be informed of group allocation the week prior to commencing patient recruitment. Each hospital will adhere to the randomised protocol for a time period until the target number of patients for each group has been met, according to the sample size calculation. Recruitment numbers will be monitored centrally by the AOANJRR and hospitals will be informed of when they have met their recruitment target. They will then crossover to the alternate treatment group, aiming for the same number of patients in each group.

Patients will be informed about the study but will not be required to individually consent to the trial, as the unit of randomisation is the hospital and both treatments represent standard practice in Australia. Patients will individually consent for the use of their data and for postoperative follow-up within the Clinical Trials Platform.

\section{Adherence}

Patients may discontinue the drug if they have an allergy or adverse event related to the drug. The study drug may be withheld if postoperative wound ooze continues

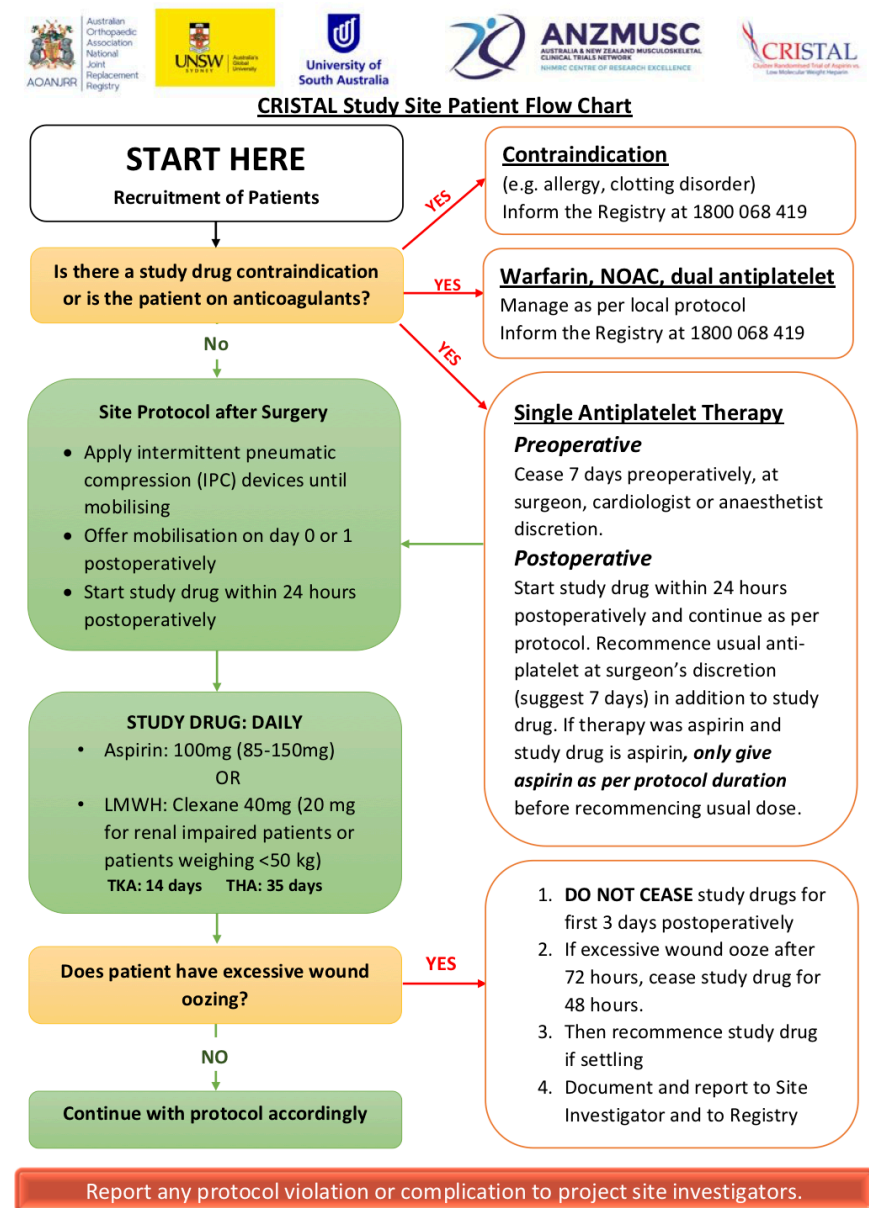

Figure 2 Patient management flow sheet. LMWH, low molecular weight heparin; NOAC, novel oral anticoagulant; THA, total hip arthroplasty; TKA, total knee arthroplasty.

beyond 72 hours postoperatively, with recommencement 48 hours later if the wound ooze has settled.

Inpatient adherence to the protocol (including drug used, dose and use of mechanical prophylaxis) during the acute care period will be determined by a 2-week chart audit of patients from each group (LMWH or aspirin) from each hospital, performed immediately after recruitment has started, independent to study investigators. Meetings will be organised with sites which have an adherence to the study drug of $<80 \%$ to discuss options to improve adherence and these sites will be re-audited prior to crossover to the alternate drug, to ensure a compliance rate of $>80 \%$ is achieved. Post-discharge adherence questions will be included in the postoperative 90-day survey.

\section{Data collection methods}

Data collection will occur preoperatively and postoperatively (at 90 days and 6 months). Table 1 outlines a timeline of data collected at each timepoint. Data collection will be achieved via patient-completed electronic surveys (via tablet, phone or computer) using direct data entry through the AOANJRR Clinical Trials Platform. Patients will be registered for the CRISTAL study at preadmission appointments and will be provided with log-in details for 


\begin{tabular}{|c|c|}
\hline Timepoint & $\begin{array}{l}\text { Data collection questions and } \\
\text { instruments }\end{array}$ \\
\hline Preoperative & $\begin{array}{l}\text { Current anticoagulation use (yes/no and } \\
\text { drug) } \\
\text { Age } \\
\text { Sex } \\
\text { Joint (hip or knee) } \\
\text { Side } \\
\text { Unilateral vs bilateral } \\
\text { Primary or revision } \\
\text { ASA grade } \\
\text { BMI } \\
\text { History of VTE } \\
\text { Oxford Hip or Knee Score } \\
\text { EQ-5D-5L } \\
\text { EQ-VAS } \\
\text { Joint pain (numeric rating scale 0-10) }\end{array}$ \\
\hline 90 days & $\begin{array}{l}\text { VTE (DVT or PE) } \\
\text { Adherence (did you use pills or injections } \\
\text { to prevent a blood clot postoperatively, for } \\
\text { how long?) } \\
\text { Current use of anticoagulants (yes/no, } \\
\text { which one) } \\
\text { Non-VTE complications (readmission, } \\
\text { readmission reason, reoperation, } \\
\text { reoperation reason, major bleeding, death) }\end{array}$ \\
\hline 6 months & $\begin{array}{l}\text { Non-VTE complications (reoperation, } \\
\text { reoperation reason, death) } \\
\text { Oxford Hip or Knee Score } \\
\text { EQ-5D-5L } \\
\text { EQ-VAS } \\
\text { Joint pain (numeric rating scale } 0-10 \text { ) }\end{array}$ \\
\hline
\end{tabular}

ASA, American Society of Anaesthesiologists; BMI, body mass index; DVT, deep venous thrombosis; EQ-5D-5L, EuroQol-5D-5L; EQ-VAS, EuroQol-visual analogue scale; PE, pulmonary embolus; VTE, venous thromboembolism.

the online data capture system. Patients can complete preoperative data entry while at the preadmission appointment (via portable devices) or online at home (using the provided log-in details).

Preoperative data collected will include demographic and operative data already captured by the AOANJRR and additional data specific to the study: anticoagulant use (online supplementary appendix 1) and patientreported pain, function and health status measures (patient-reported outcome measures (PROMs)). The following PROMs, which have been used widely in international joint registries to guide information about patient-reported outcomes post-THA and post-TKA, ${ }^{31}$ will be measured at baseline:

- The Oxford Hip and Knee Scores: these were developed to assess the outcome of hip and knee replacements and were designed to be completed by patients in order to minimise potential observer bias. Both instruments include 12 questions to assess a patient's pain and function related to their affected hip or knee. $^{27}$
- The EQ-5D: this is a standardised measure of health status developed by the EuroQoL Group in order to provide a simple, generic measure of health for clinical and economic appraisal. ${ }^{28}$ It includes five health outcome domains (mobility, self-care, usual activities, pain/discomfort and anxiety/depression) that can be summarised into a utility score. There are five descriptive sentences under each heading and patients are directed to tick one box that best describes their health on that day. The EQ-VAS addresses current health state. ${ }^{28}$

Postoperatively, patients will be followed up at 90 days and 6 months and will be prompted via email, SMS or telephone to complete postoperative surveys. To ensure minimal inconvenience, a maximum of three successful reminders will be sent to each patient. This will occur at 90 and 95 days via email and SMS and at 100 days via telephone for the 90-day postoperative survey and at 5.5 and 6 months via email and SMS and at 6.5 months via telephone for the 6-month postoperative survey. Patients will be contacted beyond 100 days and 6.5 months via telephone, if surveys remain incomplete and if initial telephone contact has not been successful. The postoperative 90-day survey will ask a series of questions regarding VTE-related and non-VTErelated complications (online supplementary appendix 2). All patients who respond 'yes' to having experienced a VTE will have this result verified by AOANJRR staff by contact with treating doctors. As a secondary measure to capture possible missed VTEs, patients will also be asked if they are still taking anticoagulant medication in the postoperative survey and those who are, will be probed for the reasons for ongoing anticoagulation. Patients who do not report having a VTE at postoperative follow-up will not have this verified routinely. However, a random subsample of 200 patients reporting an absence of VTE will have this result audited by contact with treating doctors and hospitals.

The postoperative 6-month survey will ask questions regarding reoperation (and reasons for reoperation) and will measure the above PROMs to determine change in patient-reported pain, function and health status from baseline to 6 months postoperatively in each group. NonVTE-related complications will be recorded and verified in a similar fashion at postoperative surveys.

The AOANJRR links to the National Death Index twice a year (February and September). If a patient who has participated in the CRISTAL study is flagged as deceased in the AOANJRR database, this will be transferred to the CRISTAL database and will provide data on death within 90 days and 6 months. No further contact will be made with patients or families if this occurs.

Some patients may not be able to access or use the online system to enter data, for example, older patients and nonEnglish-speaking patients. To overcome these barriers, patients will be given the option to nominate a 'proxy' (eg, family member or friend) to assist them with completing the surveys and receive reminders electronically on their behalf. Information will be collected on whether the patient had assistance to complete the postoperative survey. 


\section{Primary outcome}

The primary outcome of the trial is verified, symptomatic VTE (DVT or PE) within 90 days of HA or KA. Patients with painful, persistent leg swelling and calf tenderness postoperatively will be investigated for DVT and patients with dyspnoea, chest pain or documented hypoxia will be investigated for PE. Due to their questionable clinical relevance, routine ultrasound or venographic screening for asymptomatic DVTs will not be conducted and asymptomatic DVTs will not be included in the primary outcome for this study. ${ }^{432}$

\section{Secondary outcomes}

Secondary outcomes will include non-VTE complications (at 90 days and 6 months) and change in the abovementioned PROMs. Complications will be classified into the following groups:

- Readmission related to the original surgery or associated treatment (including bleeding and VTE related) within 90 days.

- Reason for readmission: infection, dislocation, stiffness, fracture, wound dehiscence, implant issues (loosening, migration or failure), wound bleeding, other bleeding.

- Reoperation on the same joint within 90 days and 6 months

- Type of reoperation: treatment of infection, treatment of joint bleeding or haematoma, reduction of dislocation, manipulation under anaesthesia, fracture treatment, wound repair, implant issues as above, non-joint-related surgery.

- Major bleeding events: bleeding resulting in readmission, reoperation or death, within 90 days.

- Death within 90 days and 6 months.

A cost-effectiveness analysis will be performed if aspirin is found to be inferior to LMWH. A second substudy comparing the incidence of persistent wound drainage (defined as persistent, ongoing wound drainage beyond 72 hours postoperatively) and wound disturbance (defined as wound haematoma requiring readmission, wound blistering, prolonged drainage for $>7$ days postoperatively and need for re-operation due to wound complications) between the aspirin and LMWH groups will be performed at two high volume hospitals (the Institute of Rheumatology and Orthopaedics and Fairfield Hospitals in New South Wales) participating in the CRISTAL trial. Both studies will be published as separate reports.

\section{Sample size}

A recent large cohort study of 1900 patients with THA and TKA from 19 institutions across Australia showed an incidence of symptomatic VTE within 90 days of THA and TKA of $2.6 \%$ (manuscript under preparation). A recent randomised trial of aspirin versus rivaroxaban used a minimum clinically important difference of $1 \%$, based on a survey of thromboembolism experts and orthopaedic surgeons. $^{2122}$
For the sample size calculation in the CRISTAL study, we used an estimated overall event rate of $2 \%$ (a conservative estimate based on the recent Australian cohort study and the current available literature), ${ }^{33} 34$ the same noninferiority margin of $1 \%$ from the recent randomised controlled trial $^{22}$ (an event rate of $2.5 \%$ for aspirin and $1.5 \%$ for LMWH), a power of $90 \%$ and a one-sided significance level of 0.025 . For an individual randomised trial, this yields a sample size of 4117 per treatment group or a total of 8234 patients. For a cluster randomised crossover trial, the sample size must account for correlations within clusters during the same time period (intracluster correlation) and between study periods in the same cluster (interperiod correlation). ${ }^{35}$ Assuming an intracluster correlation of 0.01 , an interperiod correlation of 0.008 and 31 clusters, the sample size required increases to 11160 patients. From each cluster, we will aim to recruit 251 registered patients from each group (a total of 15562 patients), which will allow a $27 \%$ loss to follow-up.

Due to uncertainty around the exact event rate 192122242537 and to allow for a smaller non-inferiority margin, we have constructed a sample-size table (table 2 ) to demonstrate that the trial will be adequately powered using a non-inferiority margin of $1 \%$, for an event rate up to $3 \%$ at $80 \%$ power and for an event rate up to $2 \%$ at $90 \%$ power, provided that loss to follow-up is $<17 \%$. As a secondary measure, after 1000 patients have completed the 90-day follow-up, we will obtain a preliminary symptomatic VTE rate for the whole sample and a loss to follow-up rate (without performing any comparative statistical analyses and maintaining blinding) to determine whether the estimates for the primary event rate $(2 \%)$ and loss to follow-up rate $(27 \%)$ are accurate and adjust the sample size accordingly if the primary event rate is $>3 \%$, while accounting for loss to follow-up.

\section{Statistical analysis}

The analysis for the primary objective will be limited to patients undergoing elective primary THA or TKA for a diagnosis of OA, excluding patients for whom the study drugs were contraindicated (eg, allergy or need for alternative anticoagulant-warfarin, NOAC, dual antiplatelet, for a pre-existing condition). This analysis will test between-group difference in the proportion of cases developing VTE within 90 days for non-inferiority of aspirin at a margin of $1 \%$, on an intention-to-treat basis.

The primary analysis will use cluster summary methods. ${ }^{38}$ These methods estimate the treatment effect using cluster level differences and have been shown to be appropriate for cluster randomised crossover trials with rare outcomes and the intracluster and interperiod correlation coefficients expected in this trial. ${ }^{39}$

Multiple imputation to account for missing outcome data will be investigated, using auxiliary variables gathered from routine AOANJRR data (including age, sex, baseline health, pain and function, diagnosis and surgical factors). Since VTE is rare, if prediction in the imputation models using these auxiliary variables is a problem, no imputation will be performed due to the possibility of bias. ${ }^{40}$ Since the most 
Table 2 Sample size table for the CRISTAL trial ${ }^{*} \dagger$

\begin{tabular}{|c|c|c|c|c|c|c|}
\hline Event rate in experimental & $\begin{array}{l}\text { Event rate } \\
\text { in control }\end{array}$ & $\begin{array}{l}\text { Overall } \\
\text { event rate }\end{array}$ & $\begin{array}{l}\text { Non- } \\
\text { inferiority } \\
\text { margin }\end{array}$ & $\begin{array}{l}\mathrm{N} \text { in each arm } \\
\text { (individual) }\end{array}$ & $\begin{array}{l}\text { Cluster } \\
\text { size (for } 31 \\
\text { clusters) }\end{array}$ & $\begin{array}{l}\mathrm{N} \text { total } \\
\text { (cluster } \\
\text { randomised) }\end{array}$ \\
\hline \multicolumn{7}{|l|}{ Power $=0.8$} \\
\hline 0.015 & 0.005 & 0.01 & 0.01 & 1553 & 56 & 3472 \\
\hline 0.02 & 0.01 & 0.015 & 0.01 & 2319 & 88 & 5456 \\
\hline 0.025 & 0.015 & 0.02 & 0.01 & 3076 & 123 & 7626 \\
\hline 0.03 & 0.02 & 0.025 & 0.01 & 3826 & 163 & 10106 \\
\hline 0.035 & 0.025 & 0.03 & 0.01 & 4567 & 207 & 12834 \\
\hline 0.04 & 0.03 & 0.035 & 0.01 & 5301 & 258 & 15996 \\
\hline 0.0125 & 0.005 & 0.00875 & 0.0075 & 2420 & 92 & 5704 \\
\hline 0.015 & 0.0075 & 0.01125 & 0.0075 & 3104 & 124 & 7688 \\
\hline 0.0175 & 0.01 & 0.01375 & 0.0075 & 3784 & 160 & 9920 \\
\hline 0.02 & 0.0125 & 0.01625 & 0.0075 & 4461 & 201 & 12462 \\
\hline 0.0225 & 0.015 & 0.01875 & 0.0075 & 5134 & 246 & 15252 \\
\hline \multicolumn{7}{|l|}{ Power $=0.9$} \\
\hline 0.015 & 0.005 & 0.01 & 0.01 & 2079 & 77 & 4774 \\
\hline 0.02 & 0.01 & 0.015 & 0.01 & 3103 & 124 & 7688 \\
\hline 0.025 & 0.015 & 0.02 & 0.01 & 4117 & 180 & 11160 \\
\hline 0.03 & 0.02 & 0.025 & 0.01 & 5121 & 245 & 15190 \\
\hline 0.015 & 0.0075 & 0.01125 & 0.0075 & 4154 & 182 & 11284 \\
\hline 0.0175 & 0.01 & 0.01375 & 0.0075 & 5065 & 241 & 14942 \\
\hline
\end{tabular}

*A one-sided $\alpha=0.025$ is required for a $95 \% \mathrm{Cl}$. The number of clusters is assumed to 31 , the $\mathrm{ICC}=0.01$ and the $\mathrm{IPC}=0.008$.

†Table does not account for an estimation of loss to follow-up.

ICC, intracluster correlation coefficient; IPC, intermittent pneumatic compression.

likely reason for loss to follow-up is difficulty in contacting patients postoperatively (rather than any association with treatment assignment or outcome), missing outcome data are expected to be missing completely at random, which will not cause bias in the estimates.

Secondary analyses will be performed for the primary outcome, to test for differences in treatment effect between subgroups of patients: THA only, TKA only and bilateral joint replacement. The analysis method will be the same as the primary outcome and will include an interaction term between subgroup and treatment group.

Further secondary analyses will include an extension of the primary analysis for patients undergoing all forms of HA and KA (total, revision, partial) for any indication (non-OA diagnoses) and will include patients for whom the study drug was contraindicated. This will assess the effect of implementing the protocol at a departmental (hospital) level. Other secondary analyses will include an analysis of the subcategories of VTE as the outcome; PE only, all DVT, above knee DVT only and below knee DVT only and non-VTE-related complications (death, re-operation, readmission and major bleeding rates). Cluster summary methods will be used for all secondary analyses.

If aspirin is found to be inferior to LMWH, a costeffectiveness analysis of aspirin compared with LMWH will be performed from a health system perspective. Data for resource use associated with treatments and complications will be taken from trial data within the AOANJRR. Survival at 1 year and quality of life measured using EQ-5D at baseline and 6 months will allow calculation of differences in quality-adjusted life years (QALYs) between groups. We will calculate the cost per QALY for each treatment comparison as the difference in mean costs divided by the difference in mean outcomes (quality-adjusted survival as QALYs) over the duration of the trial, using regression analysis to adjust for differences at baseline and clustering by site.

A substudy comparing rates of PWD between aspirin and LMWH groups at two sites participating in the CRISTAL trial will be performed. The analysis for this study will test the between-group difference in the proportion of cases developing PWD postoperatively, using the $\chi^{2}$ statistic and a logistic regression model, accounting for confounding patient and surgical factors (age, body mass index, sex, American Society of Anaesthesiologists grading, diabetes, rheumatoid arthritis, liver disease, cardiac disease, peripheral vascular disease, cerebrovascular disease, type of joint replacement, tourniquet use, tranexamic acid use and method of skin closure).

A detailed statistical analysis plan will be published separately to this protocol. 


\section{Data management, monitoring and sharing}

All collected data will automatically be entered in the online AOANJRR Clinical Trials Platform. Data will be stored by the AOANJRR, which is required to have highly secure data protection systems and will be managed by a separate Data Quality Committee. Data will be made available to investigators on trial completion. De-identified datasets and dictionaries will be made available for further research after trial completion and dissemination of the results via peer-review journal publication, on request from the Chief Investigator (IAH).

Given both interventions are commonly used and recommended treatments, a separate safety monitoring committee will not be established, no stopping rules will be used and no interim analysis will be performed. All outcomes (primary and secondary) will be verified by the outcome verification committee (see 'Study governance' section).

\section{Patient and public involvement}

Patients and consumers were involved in the design of the trial, the design of surveys and the choice of PROMs used in the trial. Patients and consumers also contributed to writing of the study protocol for publication.

\section{Study governance}

Separate trial management, steering and outcome verification committees have been established. The steering committee, consisting of all named authors of this protocol is responsible for final protocol approval, study oversight and approval of the principal publication. The trial management committee (IAH, SEG, VSS, SA, RSdeS, DA, GO'D, ECG, NLP) is responsible for ethics approval, liaising with sites (recruitment and maintenance) and adverse event monitoring.

The Trial is sponsored by the University of New South Wales (UNSW) and any participant claims will be responded to under UNSW's Clinical Trials insurance.

\section{Ethics and dissemination}

Any significant modifications to the trial protocol will be submitted as formal ethics amendments to lead and sitespecific ethics committees and hospitals and patients will be informed of these.

A manuscript with the results of the primary study will be published on trial completion in a peer-reviewed journal. All authors have contributed sufficiently to this study protocol and authorship of the final manuscript will be determined by the International Committee of Medical Journal Editors criteria. It is anticipated that the findings will be incorporated into clinical practice guidelines.

The trial has been endorsed by the Australia and New Zealand Musculoskeletal (ANZMUSC) Clinical Trials Network indicating its high clinical priority and quality, importance to consumers, patients, clinicians and policy makers and its potential to improve patient outcomes. The protocol manuscript adheres to the ANZMUSC governance and publication policies as will further trialrelated manuscripts.

Author affiliations

${ }^{1}$ CRISTAL Study Group, Whitlam Orthopaedic Research Centre, Ingham Institute for Applied Medical Research, University of New South Wales South Western Sydney Clinical School, Liverpool, New South Wales, Australia

${ }^{2}$ Australian Orthopaedic Association National Joint Replacement Registry, Adelaide, South Australia, Australia

${ }^{3}$ Department of Epidemiology and Preventive Medicine, School of Public Health and Preventive Medicine, Monash University, Melbourne, Victoria, Australia

${ }^{4}$ Monash Department of Clinical Epidemiology, Cabrini Institute, Melbourne, Victoria, Australia

${ }^{5}$ School of Pharmacy and Medical Sciences, University of South Australia, Adelaide, South Australia, Australia

${ }^{6}$ Department of Surgery, Epworth Healthcare, University of Melbourne, Melbourne, Victoria, Australia

${ }^{7}$ Department of Haematology, Saint George and Sutherland Clinical School, University of New South Wales, Sydney, New South Wales, Australia

${ }^{8}$ Faculty of Medicine, Saint George and Sutherland Clinical School, University of New South Wales, Sydney, New South Wales, Australia

${ }^{9}$ Monash University Centre for Health Economics, Caufield, Victoria, Australia

${ }^{10}$ South Australian Health and Medical Research Institute, Adelaide, South Australia, Australia

${ }^{11}$ Department of Intensive Care, St John of God Hospital, Subiaco, Western Australia, Australia

${ }^{12}$ Department of Epidemiology and Preventive Medicine, School of Public Health and Preventive Medicine, Monash University, Melbourne, Victoria, Austria

${ }^{13}$ Musculoskeletal Australia, Melbourne, Victoria, Australia

${ }^{14}$ Institute for Musculoskeletal Health, The University of Sydney School of Public Health, Sydney, New South Wales, Australia

\section{Twitter llana N Ackerman @arthritis}

Contributors All authors listed have contributed to this protocol and the International Committee of Medical Journal Editors guidelines were consulted for determining authorship. VSS, IAH, RB, SEG, SA, AHar, JMN, RSdeS, NLP, BHC, INA, ECG, ML, SW, OC, DA, GO'D and LK were responsible for the planning of the trial, protocol development and writing, $\mathrm{MC}$ and AHan were consulted as patient consumers for survey development and NP and LK were responsible for the sample size calculation and the description of the statistical analyses to be used. All authors reviewed the final version of this manuscript prior to submission.

Funding The study is being funded by a 4-year Medical Research Futures Fund (Australian Federal Government) grant.

Disclaimer The Standard Protocol Items: Recommendations for Interventional Trials statement for the reporting of clinical trial protocols was used to guide the development of this protocol.

Competing interests None declared.

Patient consent for publication Not required.

Ethics approval Ethics approval has been granted by the Sydney Local Health District (Royal Prince Alfred Zone) Human Research and Ethics Committee, which is a lead ethics committee in Australia (approval number X18-0424). Site-specific approvals for each participating hospital have also been granted from the following ethics committees: Calvary John James Memorial Hospital Australian Capital Territory (3-2019 CRISTAL), Mid-North Coast Local Health District New South Wales (NSW—SSA/19/NCC/41), South Western Sydney Local Health District (NSW, SSA/10/LP0OL/22), Sydney Local Health District (NSW, SSA/19/RPAH/12, SSA/18/ RPAH/762), Ramsay Hospital Research Foundation (NSW, HREC/18/RPAH/603), South Eastern Sydney Local Health District (NSW, 19/G/028, 18/G/338), Nepean Blue Mountains Local Health District (NSW, SSA/19/NEPAN/11), Northern Sydney Local Health District (NSW, RESP/19/027, RESP/19/028), Metro North Hospital and Health Service (Queensland, HREC/18/RPAH/603), University of South Australia (SA-201215), Calvary Healthcare Adelaide (SA, 19-CHREC-F001), Southern Adelaide Local Health Network (SA, HREC/18/RPAH/603), Bendigo Health Victoria (SSA/48255/BHCG-2019), Barwon Health (18/246), Peninsula Health Victoria (SSA/48255/PH-2019), Western Health Victoria (48255), St John of God Healthcare Victoria (1540), South Metropolitan Health Western Australia (WA - Western Australia, RGS0000001358), North Metropolitan Health (WA-RGS0000001358), 
Sir Charles Gardiner Hospital (WA—RGS0000001358) and Launceston General Hospital Tasmania (H0017903).

Provenance and peer review Not commissioned; externally peer reviewed.

Open access This is an open access article distributed in accordance with the Creative Commons Attribution Non Commercial (CC BY-NC 4.0) license, which permits others to distribute, remix, adapt, build upon this work non-commercially, and license their derivative works on different terms, provided the original work is properly cited, appropriate credit is given, any changes made indicated, and the use is non-commercial. See: http://creativecommons.org/licenses/by-nc/4.0/.

\section{ORCID iDs}

Verinder Singh Sidhu http://orcid.org/0000-0001-6511-1383

Ilana N Ackerman http://orcid.org/0000-0002-6028-1612

\section{REFERENCES}

1 AOA. Australian Orthopaedic Association national joint replacement registry annual report, 2017.

2 An VVG, Phan K, Levy YD, et al. Aspirin as Thromboprophylaxis in Hip and Knee Arthroplasty: A Systematic Review and Meta-Analysis. J Arthroplasty 2016;31:2608-16.

3 Mirkazemi C, Bereznicki LR, Peterson GM. Comparing Australian orthopaedic surgeons' reported use of thromboprophylaxis following arthroplasty in 2012 and 2017. BMC Musculoskelet Disord 2019;20:57.

4 Falck-Ytter Y, Francis CW, Johanson NA, et al. Prevention of VTe in orthopedic surgery patients: antithrombotic therapy and prevention of thrombosis, 9th ED: American College of chest physicians evidence-based clinical practice guidelines. Chest 2012;141:e27 8S-325.

5 Mont MA, Jacobs JJ, Boggio LN, et al. Preventing venous thromboembolic disease in patients undergoing elective hip and knee arthroplasty. J Am Acad Orthop Surg 2011;19:768-76.

6 NICE. Venous thromboembolism in over 16s: reducing the risk of hospital-acquired deep vein thrombosis or pulmonary embolism, 2018.

7 Jenny J-Y, Pabinger I, Samama CM, et al. European guidelines on perioperative venous thromboembolism prophylaxis: aspirin. Eur $J$ Anaesthesiol 2018;35:123-9.

8 Russell RD, Huo MH. Apixaban and rivaroxaban decrease deep venous thrombosis but not other complications after total hip and total knee arthroplasty. J Arthroplasty 2013;28:1477-81.

9 Nieto JA, Espada NG, Merino RG, et al. Dabigatran, rivaroxaban and apixaban versus enoxaparin for thomboprophylaxis after total knee or hip arthroplasty: pool-analysis of phase III randomized clinical trials. Thromb Res 2012;130:183-91.

10 Hull R, Raskob G, Pineo G, et al. A comparison of subcutaneous low-molecular-weight heparin with warfarin sodium for prophylaxis against deep-vein thrombosis after hip or knee implantation. N Engl $J$ Med 1993;329:1370-6.

11 Turun S, Banghua L, Yuan Y, et al. A systematic review of rivaroxaban versus enoxaparin in the prevention of venous thromboembolism after hip or knee replacement. Thromb Res 2011:127:525-34.

12 NHMRC. National Health and Medical Research Council. Clinical Practice Guideline for the Prevention of Venous Thromboembolism Rescinded, 2009.

13 Adam SS, McDuffie JR, Lachiewicz PF, et al. Comparative effectiveness of new oral anticoagulants and standard thromboprophylaxis in patients having total hip or knee replacement: a systematic review. Ann Intern Med 2013;159:275-84.

14 Gómez-Outes A, Terleira-Fernández Al, Suárez-Gea ML, et al. Dabigatran, rivaroxaban, or apixaban versus enoxaparin for thromboprophylaxis after total hip or knee replacement: systematic review, meta-analysis, and indirect treatment comparisons. BMJ 2012;344:e3675.

15 Cao YB, Zhang JD, Shen H, et al. Rivaroxaban versus enoxaparin for thromboprophylaxis after total hip or knee arthroplasty: a meta-analysis of randomized controlled trials. Eur J Clin Pharmacol 2010;66:1099-108.

16 Cohen A, Drost P, Marchant N, et al. The efficacy and safety of pharmacological prophylaxis of venous thromboembolism following elective knee or hip replacement: systematic review and network meta-analysis. Clin Appl Thromb Hemost 2012;18:611-27.

17 Harenberg J, Marx S, Dahl OE, et al. Interpretation of endpoints in a network meta-analysis of new oral anticoagulants following total hip or total knee replacement surgery. Thromb Haemost 2012;108:903-12.

18 Neumann I, Rada G, Claro JC, et al. Oral direct factor Xa inhibitors versus low-molecular-weight heparin to prevent venous thromboembolism in patients undergoing total hip or knee replacement: a systematic review and meta-analysis. Ann Intern Med 2012:156:710-9.

19 Wilson DGG, Poole WEC, Chauhan SK, et al. Systematic review of aspirin for thromboprophylaxis in modern elective total hip and knee arthroplasty. Bone Joint J 2016;98-B:1056-61.

20 Drescher FS, Sirovich BE, Lee A, et al. Aspirin versus anticoagulation for prevention of venous thromboembolism major lower extremity orthopedic surgery: a systematic review and meta-analysis. $J$ Hosp Med 2014;9:579-85.

21 Anderson DR, Dunbar MJ, Kahn SR. Aspirin versus low-molecularweight heparin after total hip arthroplasty. Ann Intern Med 2013;159:502-3.

22 Anderson DR, Dunbar M, Murnaghan J, et al. Aspirin or rivaroxaban for VTe prophylaxis after hip or knee arthroplasty. N Engl J Med 2018;378:699-707.

23 Bozic KJ, Vail TP, Pekow PS, et al. Does aspirin have a role in venous thromboembolism prophylaxis in total knee arthroplasty patients? $J$ Arthroplasty 2010;25:1053-60.

24 Jameson SS, Baker PN, Charman SC, et al. The effect of aspirin and low-molecular-weight heparin on venous thromboembolism after knee replacement: a non-randomised comparison using national joint registry data. J Bone Joint Surg Br 2012;94:914-8.

25 Jameson SS, Charman SC, Gregg PJ, et al. The effect of aspirin and low-molecular-weight heparin on venous thromboembolism after hip replacement: a non-randomised comparison from information in the National joint registry. J Bone Joint Surg Br 2011;93:1465-70.

26 Molnar RB, Jenkin DE, Millar MJ, et al. The Australian arthroplasty thromboprophylaxis survey. J Arthroplasty 2012;27:173-9.

27 Murray DW, Fitzpatrick R, Rogers K, et al. The use of the Oxford hip and knee scores. J Bone Joint Surg Br 2007;89:1010-4.

28 EuroQol Group. EuroQol--a new facility for the measurement of health-related quality of life. Health Policy 1990;16:199-208.

29 Muñoz-Venturelli P, Arima H, Lavados P, et al. Head Position in Stroke Trial (HeadPoST)--sitting-up vs lying-flat positioning of patients with acute stroke: study protocol for a cluster randomised controlled trial. Trials 2015;16:256.

30 van Duijn PJ, Verbrugghe W, Jorens PG, et al. The effects of antibiotic cycling and mixing on antibiotic resistance in intensive care units: a cluster-randomised crossover trial. Lancet Infect Dis 2018;18:401-9.

31 Rolfson O, Bohm E, Franklin P, et al. Patient-Reported outcome measures in arthroplasty registries report of the patient-reported outcome measures Working group of the International Society of arthroplasty registries Part II. recommendations for selection, administration, and analysis. Acta Orthop 2016;87:9-23.

32 Wang CJ, Wang JW, Weng LH, et al. Outcome of calf deep-vein thrombosis after total knee arthroplasty. J Bone Joint Surg $\mathrm{Br}$ 2003;85:841-4.

33 Kulshrestha V, Kumar S. DVT prophylaxis after TKA: routine anticoagulation vs risk screening approach - a randomized study. $J$ Arthroplasty 2013;28:1868-73.

34 Bawa H, Weick JW, Dirschl DR, et al. Trends in deep vein thrombosis prophylaxis and deep vein thrombosis rates after total hip and knee arthroplasty. J Am Acad Orthop Surg 2018;26:698-705.

35 Giraudeau B, Ravaud P, Donner A. Sample size calculation for cluster randomized cross-over trials. Stat Med 2008;27:5578-85.

36 Kelly T-L, Pratt N. A note on sample size calculations for cluster randomised crossover trials with a fixed number of clusters. Stat Med 2019;38:3342-5.

37 Cimminiello C, Prandoni P, Agnelli G, et al. Thromboprophylaxis with enoxaparin and direct oral anticoagulants in major orthopedic surgery and acutely ill medical patients: a meta-analysis. Intern Emerg Med 2017;12:1291-305.

38 Turner RM, White IR, Croudace T, et al. Analysis of cluster randomized cross-over trial data: a comparison of methods. Stat Med 2007;26:274-89.

39 Forbes $\mathrm{AB}$, Akram M, Pilcher $\mathrm{D}$, et al. Cluster randomised crossover trials with binary data and unbalanced cluster sizes: application to studies of near-universal interventions in intensive care. Clin Trials 2015;12:34-44.

40 White IR, Daniel R, Royston P. Avoiding bias due to perfect prediction in multiple imputation of incomplete categorical variables. Comput Stat Data Anal 2010;54:2267-75. 
Correction: CRISTAL: protocol for a cluster randomised, crossover, non-inferiority trial of aspirin compared to low molecular weight heparin for venous thromboembolism prophylaxis in hip or knee arthroplasty, a registry nested study

Sidhu VS, Graves SE, Buchbinder R, et al. CRISTAL: protocol for a cluster randomised, crossover, non-inferiority trial of aspirin compared to low molecular weight heparin for venous thromboembolism prophylaxis in hip or knee arthroplasty, a registry nested study. BMJ Open 2019;9:e031657. doi: 10.1136/bmjopen-2019-031657

This article was previously published with an error.

The second paragraph under the 'Study Governance' section on the eighth page has been updated from:

The AOANJRR has overall responsibility for the study. The AOANJRR is owned by the Australian Orthopaedic Association (AOA) and is funded by the Australian Federal Government. The AOA holds General Liability insurance cover and compensation will be provided to any patient who suffers harm from trial participation. Proof of insurance cover has been submitted to all ethics committees approving the trial.

to:

The Trial is sponsored by the University of New South Wales (UNSW) and any participant claims will be responded to under UNSW's Clinical Trials insurance.

Open access This is an open access article distributed in accordance with the Creative Commons Attribution Non Commercial (CC BY-NC 4.0) license, which permits others to distribute, remix, adapt, build upon this work non-commercially, and license their derivative works on different terms, provided the original work is properly cited, appropriate credit is given, any changes made indicated, and the use is non-commercial. See: http://creativecommons.org/licenses/by-nc/4.0/.

(c) Author(s) (or their employer(s)) 2022. Re-use permitted under CC BY-NC. No commercial re-use. See rights and permissions. Published by BMJ.

BMJ Open 2022;12:e031657corr1. doi:10.1136/bmjopen-2019-031657corr1

Check for updates 